\title{
Measurement of the Binding Energies of the Organic-Metal Perylene-Teracarboxylic- Dianhydride/Au(111) Bonds by Molecular Manipulation Using an Atomic Force Microscope
}

\author{
C. Wagner, ${ }^{1,2, *}$ N. Fournier, ${ }^{1,2}$ F. S. Tautz, ${ }^{1,2}$ and R. Temirov ${ }^{1,2}$ \\ ${ }^{1}$ Peter Grünberg Institut (PGI-3), Forschungszentrum Jülich, 52425 Jülich, Germany \\ ${ }^{2}$ Jülich Aachen Research Alliance (JARA), Fundamentals of Future Information Technology, 52425 Jülich, Germany
} (Received 14 March 2012; published 16 August 2012)

\begin{abstract}
Based on single molecule manipulation experiments in a combined scanning tunneling microscope/ frequency modulated atomic force microscope, we quantify the individual binding energy contributions to an organic-metal bond experimentally. The method allows the determination of contributions from, e.g., local chemical bonds, metal-molecule hybridization, and van der Waals interactions, as well as the total adsorption energy.
\end{abstract}

DOI: 10.1103/PhysRevLett.109.076102

PACS numbers: 68.37.Ef, 68.37.Ps, 82.37.Gk

The bonding of large organic adsorbates to metal surfaces is subject of an intense research effort [1-6]. If the molecule has functional groups, one may expect different bonding channels to contribute to the overall bonding of the molecule to the surface. An example for such a multifunctional bond is that of the well-studied model molecule 3,4,9,10-perylene-teracarboxylic-dianhydride (PTCDA) to noble metal (111)-surfaces. According to the established picture, derived mainly from structural and electronic structure data, the PTCDA-metal bond consists of three attractive components [4,7-13]: firstly, local bonds of the reactive carboxylic oxygen atoms $\left(\mathrm{O}_{\text {carb }}\right)$ to metal atoms in the surface; secondly, weak chemisorption of the perylene core due to hybridization of delocalized molecular and metal states; and thirdly, the van der Waals interaction. The theoretical description of such multifunctional bonds presents a challenge because individual contributions may compete against or reinforce each other depending on the situation at hand. Moreover, the van der Waals interaction is only now being included into density functional theory (DFT) [12,14-17], and there is still some uncertainty regarding the reliability of the various approaches [18]. In this situation, it would be helpful if the various contributions to the total energy of a multifunctional bond could be determined experimentally.

In this Letter, we present an experimental method which allows the partitioning of the overall bonding energy between different bonding channels and apply it to PTCDA/ $\mathrm{Au}(111)$. The approach is based on molecular manipulation in a combined scanning tunneling microscope/ frequency modulated atomic force microscope (STM/ FM-AFM) with a qPlus sensor (cf. Fig. 1) [19]. STM is used for high resolution imaging and location of the functional group at which to dock the tip to the molecule, and FM-AFM is used to measure the junction stiffness $d F_{z} / d z$ [20] while manipulating the molecule off the surface by tip retraction ( $z=$ vertical tip coordinate) [21-23]. Because the stiffness of the surface-molecule-tip junction is measured throughout the complete removal process, the strength of all interaction potentials constituting the PTCDA-Au(111) bond is probed, albeit in a tangled manner. To disentangle the various contributions, we use a procedure which is based on the hypothesis that the $\left(d F_{z} / d z\right)(z)$ curve contains enough information to retrieve the quantitative shape of all relevant interaction potentials. This hypothesis is fully borne out by our results. The retrieval, discussed in detail below, proceeds by firstly, parameterizing the various bonding channels of the molecule-substrate interaction with generic potentials of sufficient generality, secondly, simulating the junction stiffness $d F_{z} / d z$ based on these generic potentials throughout the whole manipulation process, and thirdly, fitting the simulated junction stiffness to the experiment, thereby extracting the correct potential parameters, from which the desired partitioning can finally be calculated.

The experiments are performed with a commercial STM/FM-AFM (CREATEC) operated at $5 \mathrm{~K}$ in ultrahigh

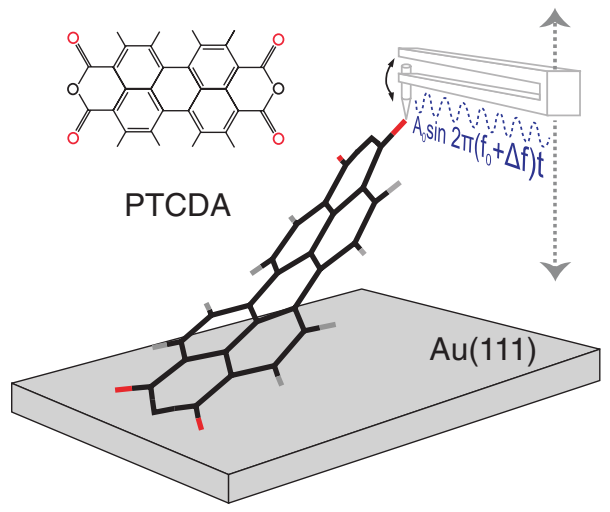

FIG. 1 (color). Scheme of the experimental setup. A single PTCDA molecule on $\mathrm{Au}(111)$ is contacted by a tip attached to a qPlus sensor. The oscillating sensor is retracted from and approached to the surface [28]. In this way, the molecule is repeatedly detached from the surface and brought back. Changes in the resonance frequency of the sensor reflect changes in the stiffness of the junction. 
vacuum. The $15 \mu \mathrm{m}$ diameter PtIr tip wire of the qPlus sensor was cut and sharpened using a focused ion beam. The tip was finally prepared at $5 \mathrm{~K}$ by indentation into the clean $\mathrm{Au}(111)$ surface. The frequency of the qPlus sensor was $f_{0}=30311 \mathrm{~Hz}$, its spring constant $k=1800 \mathrm{~N} \mathrm{~m}^{-1}$ [19]. The surface was prepared using $\mathrm{Ar}^{+}$sputtering and annealing in ultrahigh vacuum. The molecules were deposited from a home-built Knudsen cell. Single molecules were prepared by detaching them from the molecular islands with the STM/FM-AFM tip in STM mode. The contact between the tip and one of the $\mathrm{O}_{\text {carb }}$ atoms of PTCDA was established according to the procedure described earlier [20-22]. Note that during contacting and throughout the experiment the tip is vibrating with an amplitude of $\approx 0.2 \AA$ [24].

Having established the contact, the tip is retracted vertically from the surface by $27 \AA$, lifting the molecule and fully breaking the molecule-surface bond. Then, the tip is approached back to the surface, restoring the bond between PTCDA and $\mathrm{Au}(111)$. In each of the experiments, the retraction-approach cycle is repeated 10 times while $d F_{z} / d z(z)=-\left(2 k_{0} / f_{0}\right) \Delta f(z)$ [19] is recorded. As Fig. 2 (a) shows, the gradual breaking of the molecule-surface bond is reversible and reproducible. The dramatic improvement in reproducibility over previous work [20] is attributed to the low corrugation of the adsorption potential of PTCDA on $\mathrm{Au}(111)$. Hence, during tip retraction the lower end of the molecule slides smoothly over the surface, while on $\operatorname{Ag}(111)$ its motion is more abrupt and thus less controlled [20].

In order to extract the desired information from the histogram in Fig. 2(a), we model the PTCDA-metal bond by generic potentials for the following four moleculesubstrate interaction channels that are qualitatively well established for PTCDA on noble metal surfaces [4]: (1) the local chemical bonds between the $\mathrm{O}_{\text {carb }}$ atoms and the surface, (2) the chemical interaction due to hybridization of the delocalized molecular orbitals with substrate states, (3) the dispersion attraction, and finally (4) the Pauli repulsion. The delocalized chemical and dispersion interactions are split into interactions of individual atoms in the molecule with the surface. In this way, three atom-surface interaction potentials are defined for each atom in PTCDA (Table I). These potentials are parametrized by a set $P$ of nine parameters [25]. There is no surface corrugation in our model, which is a good approximation for PTCDA/ $\mathrm{Au}(111)$ [26].

The internal mechanical properties of the molecule itself are described by a standard molecular-mechanics model including covalent bonds, valence and dihedral angles, and van der Waals and electrostatic interactions. Because the molecule is strongly distorted during the lift-off [23] and because the distortion determines the precise way in which the molecule-substrate interaction potentials are sampled in the experiment, the correct description of the internal
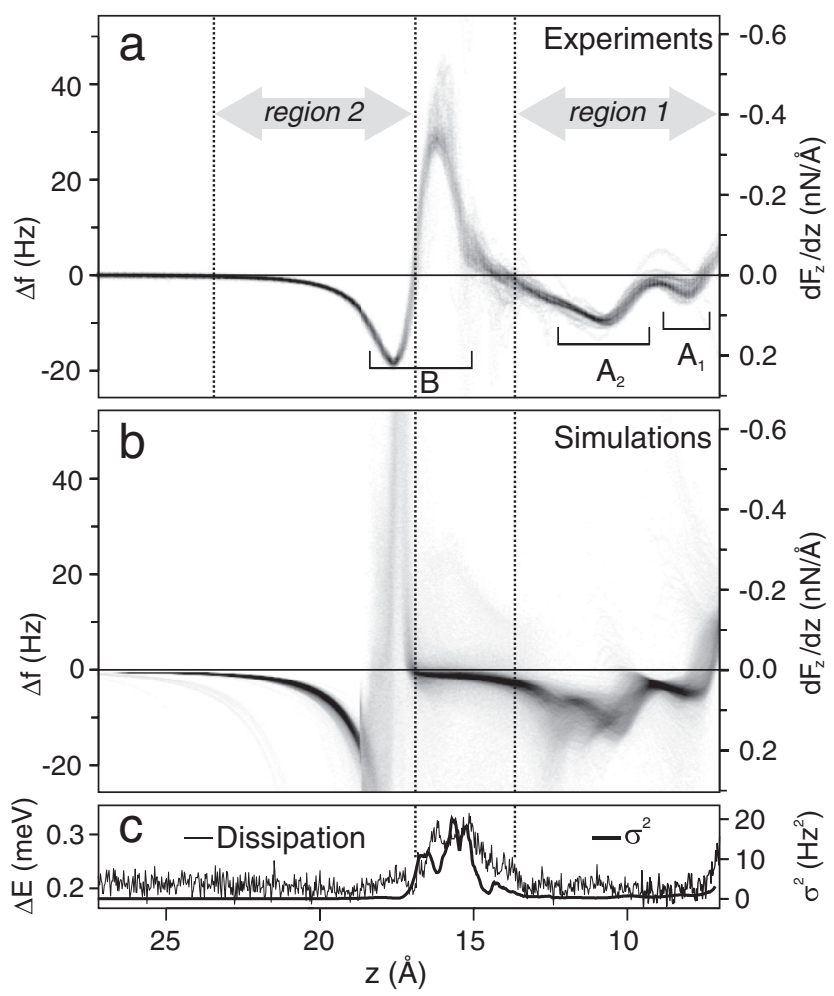

FIG. 2. (a) Histogram $(\sqrt{N})$ of $226 \Delta f$ traces acquired during lifting and lowering of PTCDA. All curves have been aligned at feature $B$. The $\Delta f$ signal during tip approach was subtracted [20]. The $z$ axis is shifted to match the simulated curves in panel (b). Dotted lines mark regions 1 and 2 within which the correspondence between experiment and simulation is calculated (reduced $\chi^{2}$ ). Distinct features of the histogram are labeled $A_{1}$, $A_{2}$, and $B$. (b) Histogram $(\sqrt{N})$ of $4166 \Delta f$ traces simulated with different parameter sets $P$ that have passed the preselection criteria (see text). (c) The energy dissipation of the qPlus sensor as well as the experimental variance $\sigma^{2}$ show a peak around $z=16 \AA$ (see text).

mechanics of PTCDA is essential. We have therefore optimized the set $Q$ of 34 force field parameters for the internal mechanics of PTCDA by fitting them to DFT calculations [BLYP/6-31(d)] [27] of molecular geometries that correspond to the 108 vibrational modes of a free PTCDA molecule. The DFT energies for the different geometries are reproduced with an average deviation of $18 \%$ by our

TABLE I. Atom-surface potentials used to describe the PTCDA-Au(111) interaction. $z$ denotes the atom height above the surface. Superscript $C$ refers to all atoms except $\mathrm{O}_{\text {carb }}$, $\mathrm{O}$ to $\mathrm{O}_{\text {carb }}$.

\begin{tabular}{lc}
\hline \hline Potential & Parameters (set $P$ ) \\
\hline$V_{\text {Pauli }}=D_{p} \exp \left(-A_{p} z\right)$ & $D_{p}^{C}, D_{p}^{O}, A_{p}^{C}, A_{p}^{O}$ \\
$V_{\text {chem }}=-D_{c} \exp \left(-A_{c} z\right)$ & $D_{c}^{C}, D_{c}^{O}, A_{c}^{C}, A_{c}^{O}$ \\
$V_{v d W}=-D_{v d W} z^{-3}$ & $D_{v d W}$ \\
\hline \hline
\end{tabular}


force field. Details of the fitting procedure are given in the Supplemental Material [28].

The thus specified potentials allow the calculation of the total energy of the system

$$
E_{t}(P, z)=E_{\text {mol-sub }}(P, z)+E_{\text {intra }}(z)
$$

as a function of the tip coordinate $z$ that parametrizes the equilibrium junction geometry, including the molecular distortion. The equilibrium geometry for each $z$ is determined by retracting the tip vertically (as in experiment) in steps of $\Delta z=0.25 \mathrm{pm}$ and relaxing the molecular geometry with a force tolerance of $5 \times 10^{-4} \mathrm{eV} / \AA$. For each $z$, $E_{\text {mol-sub }}(P, z)$ is obtained by summing the potentials of Table I for each atom in the molecule. $E_{\text {intra }}(z)$ is the energy of the distorted molecule in the junction, calculated with force field parameters $Q$ and the molecule-tip bond [29]. The frequency shift of the qPlus sensor is calculated as described in Ref. [30]:

$$
\Delta f(P, z)=\frac{f_{0}}{\pi k_{0} A^{2}} \int_{-A}^{A} \frac{d^{2} E_{t}(P, z-q)}{d z^{2}} \sqrt{A^{2}-q^{2}} d q .
$$

The convolution with a semicircle simulates the experimental tip oscillation amplitude of $A=0.2 \AA$.

The task is to find the parameter set $P$ which, if inserted into Eqs. (1) and (2), reproduces the experimental $\Delta f$ curve in Fig. 2(a). Since each set $P$ requires a separate simulation of the complete manipulation process (which is costly in terms of CPU time), a fast preselection routine is employed which identifies promising parameter sets $P$ and discards the rest [28]. From a total of $10^{8}$ parameter sets that were randomly generated by a Monte Carlo algorithm [28], we have selected 4166 sets $P$ for which full simulations were carried out.

A histogram of the simulated $\Delta f(P, z)$ curves [Eq. (2)] for the 4166 sets $P$ is shown in Fig. 2(b). Evidently, our generic potentials simulate the experiment very well. The features $A_{1}, A_{2}$, and $B$ are reproduced with remarkable accuracy [31]. There is, however, a small $z$ shift of $\approx 1 \AA$ between experiment and simulation for $z>15 \AA$. Moreover, the experimental $\Delta f$ peak $B$ at $z=16 \AA$ is much wider than its simulated counterpart. We suggest that both the $z$ shift and the broadening of the experimental $\Delta f$ peak are due to the finite stiffness of the Au covered PtIr tip resulting in measurable relaxations that occur while lifting the molecule. Additionally, the spurious vertical forces that occur when the lower end of the molecule moves laterally on a corrugated surface also contribute to the broadening of the $\Delta f$ peak. The occurrence of such motion is indicated by an increase in dissipation around $16 \AA$ [Fig. 2(c)]. Both of the described effects can, in principle, be simulated by including a tip of finite stiffness that oscillates in the direction perpendicular to the surface in the simulation of the molecule lifting process. The results of such simulations will be discussed in the forthcoming publication. Here we account for both effects
( $z$ shift and $B$ broadening) by neglecting the experimental data between 13.7 and $16.9 \AA$ when quantifying the correspondence between individual simulations and the averaged experiment by calculating a reduced $\chi^{2}$. Because of the discontinuity in the experimental $z$ scale, regions 1 and 2 [as marked in Fig. 2(a)] are aligned separately with each simulated curve to calculate $\chi^{2}$.

We now examine the best parameter sets $P$ with the lowest $\chi^{2}$ values. In Fig. 3(a), $\chi^{2}$ is plotted versus the total binding energy of PTCDA for all sets $P$ with $\chi^{2}<3$. While the best fit to the experiment is found for a parameter set $P^{*}$ with $E_{\mathrm{bind}}^{*}=2.6 \mathrm{eV}$, the distribution of points in Fig. 3(a) yields a binding energy of $E_{\text {bind }}=(2.5 \pm 0.1) \mathrm{eV}$ for PTCDA on $\mathrm{Au}(111)$. The $\Delta f(z)$ curve that was simulated using set $P^{*}$ is shown in Fig. 4(a) [28]. We note the excellent fit between the experimental $\Delta f(z)$ and the calculated $\Delta f\left(P^{*}, z\right)$ (apart from the issue of the peak width discussed above).

To identify the nature of the PTCDA-Au(111) bond, we plot the contributions of the different potentials in Figs. 3(b) and 3(c). Here, each set $P$ is represented by six points with identical $\chi^{2}$. From the plots a clear picture emerges. While the van der Waals attraction is in the range
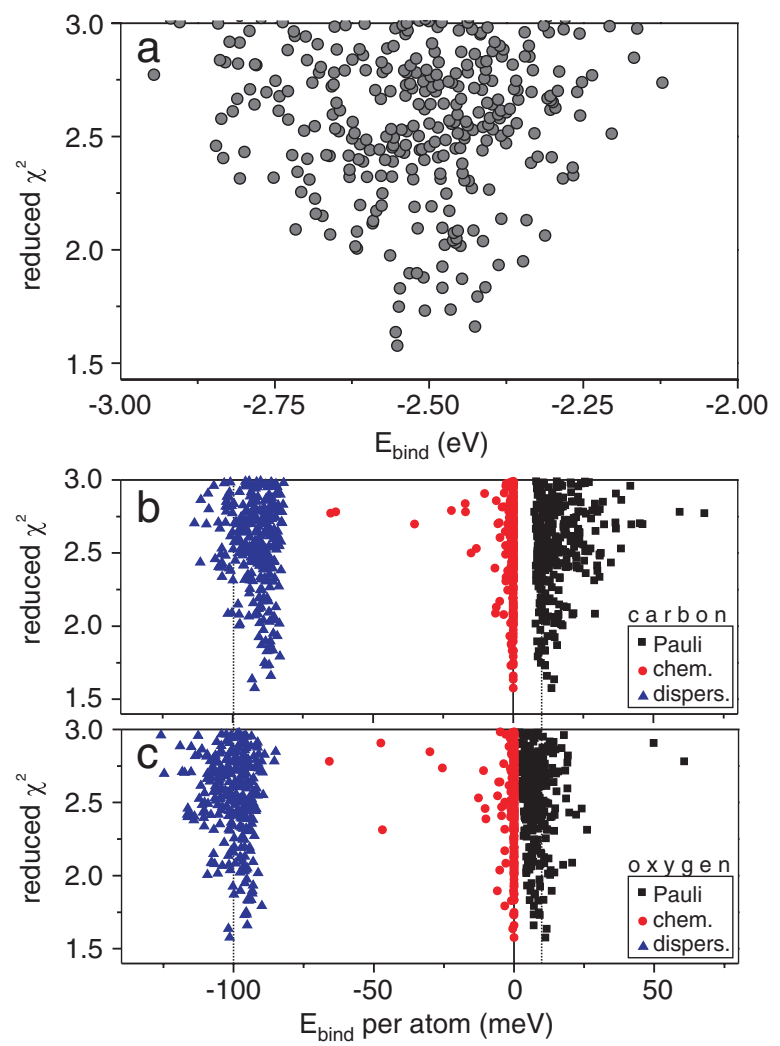

FIG. 3 (color). (a) Plot of the correspondence between individual simulations and experiment (reduced $\chi^{2}$ ) versus the adsorption energy of PTCDA for all sets $P$ with $\chi^{2}<3$. (b) and (c) Plots of $\chi^{2}$ versus the energy contributions of the different potentials (and different types of atoms). "Carbon" stands for all atoms except $\mathrm{O}_{\text {carb }}$. Dotted lines are a guide for the eye. 

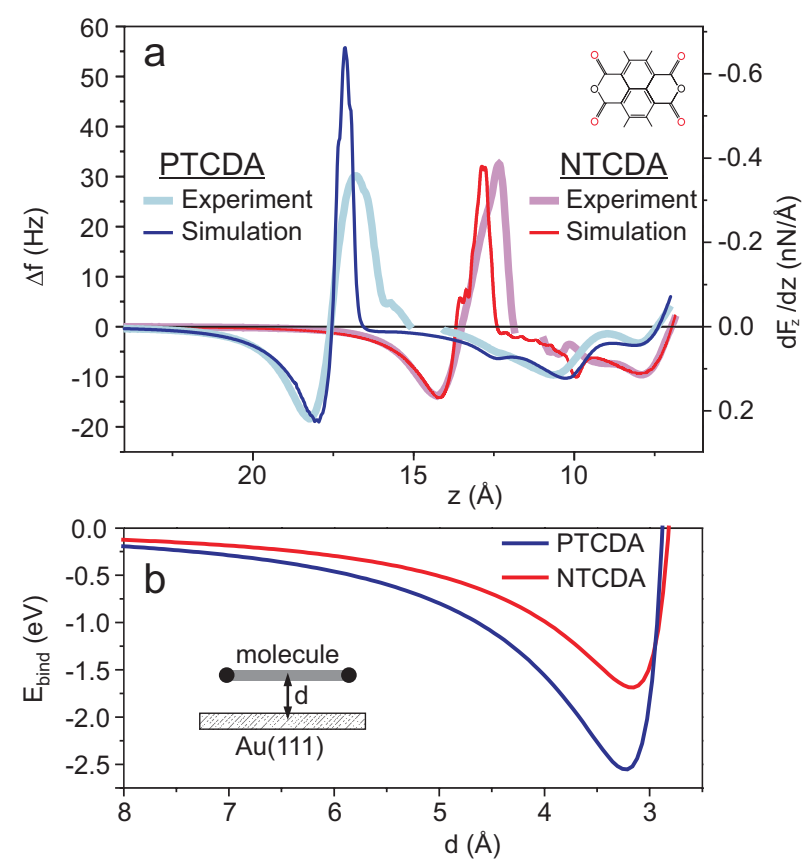

FIG. 4 (color). (a) Comparison between simulated and experimental $\Delta f$ curve for PTCDA and NTCDA on Au(111). Parameter set $P^{*}$, derived for PTCDA (see text), was used for both simulations. The experimental curves are cut in the region of an instable junction (Fig. 2), and both parts are separately aligned with the simulation. (b) Binding potential as a function of moleculesubstrate separation calculated with set $P^{*}$ for PTCDA and NTCDA. The molecule is relaxed on the surface and subsequently moved to different heights without further relaxation.

of $100 \mathrm{meV}$ for $\mathrm{C}$ as well as $\mathrm{O}_{\text {carb }}$ atoms, neither of the two species has any chemical interactions with the $\mathrm{Au}(111)$ surface. The fact that $\mathrm{O}_{\text {carb }}$ atoms tend to bind slightly stronger is due to their smaller distance $(\approx 0.1 \AA$ less $)$ to the surface in the equilibrium geometry. To get the net adsorption energy, the (positive) Pauli repulsion of $\approx 10 \mathrm{meV}$ has to be added. Comparing our result to theory, we find a good correspondence insofar as also $a b$ initio calculations, as well as other spectroscopic methods (ultraviolet photoelectron spectroscopy, x-ray standing wave, etc.), suggest pure physisorption of PTCDA on $\mathrm{Au}(111)$ $[4,10,32]$. However, our experiment yields a significantly higher adsorption energy than predicted from calculations $(\approx 2.0 \mathrm{eV}[18,26])$. This indicates an underestimation of dispersion interaction in the (semiempirically corrected) DFT method(s) used. Parameter sets $P$ which yield total binding energies in the range of $2.0 \mathrm{eV}$ are clearly inconsistent with our experimental result [Fig. 3(a)] and, hence, can be discarded. Recent DFT calculations which include dielectric screening within the substrate yield an energy of $2.4 \mathrm{eV}$, which is close to our experimental result [33].

To validate our method further and to check whether the parameters obtained are meaningful beyond the fitted example of PTCDA on $\mathrm{Au}(111)$, we use the parameter sets $P^{*}$ and $Q$ to calculate the frequency shift of the smaller
NTCDA (1,4,5,8-naphthalene-tetracaboxylic-dianhydride) molecule during lift-off from $\mathrm{Au}(111)$ [also NTCDA is known to physisorb on $\mathrm{Au}(111)$ [32]]. The comparison between simulation and experiment is shown in Fig. 4(a), showing an excellent agreement. Note that this agreement for NTCDA is not the result of any fitting but just a consequence of the universal character of our potentials. The potentials, once fitted properly to yield the correct parameter set $P^{*}$, hence have predictive power for systems which exhibit similar physics. The bonding energy of NTCDA turns out to be $1.7 \mathrm{eV}$ (compared to $1.3 \mathrm{eV}$ from DFT calculations [26]).

In conclusion, in this Letter we have reported the- to our knowledge-first analysis which allows the quantitative identification of different bonding channels of large organic adsorbates on the basis of experimental data alone. As a result of this analysis, the precise shape of the total binding potential can be determined. As an example, we show the van der Waals-like potential that results if the distance between a flat and fully relaxed PTCDA molecule and the $\mathrm{Au}(111)$ surface is varied [Fig. 4(b)]. The resulting curves can be compared to $a b$ initio calculations. Moreover, the method reported in this Letter represents a novel way to measure the adsorption energy of molecular adsorbates on a single-molecule level. Note that this method is also applicable in cases where the determination of the adsorption energy by thermal desorption spectroscopy is impossible because molecules decompose before desorbing. In the present Letter, we have carried out the experiments and the corresponding analysis for an adsorbate-substrate combination which exhibits a small lateral corrugation potential to demonstrate the principle. However, we anticipate that our approach is also applicable to more strongly corrugated substrates, if combined with a customized tip retraction trajectory [20] which minimizes sliding as much as possible.

We gratefully acknowledge T. Lippert, N. Attig, O. Büchner, and W. Homberg (Forschungszentrum Jülich) who have supported us with CPU time on JUDGE. We thank D. Park and J. Mayer from RWTH Aachen for help with the focused ion beam. R. T. thanks the HelmholtzGemeinschaft for financial support for his Young Investigator Research Group. This work has been supported by the Deutsche Forschungsgemeinschaft under projects TA244/5-1 and TA244/5-2.

*Corresponding author: c.wagner@fz-juelich.de

[1] F. Rosei, M. Schunack, Y. Naitoh, P. Jiang, A. Gourdon, E. Laegsgaard, I. Stensgaard, C. Joachim, and F. Besenbacher, Prog. Surf. Sci. 71, 95 (2003).

[2] G. Witte and C. Wöll, J. Mater. Res. 19, 1889 (2004).

[3] J. V. Barth, Annu. Rev. Phys. Chem. 58, 375 (2007).

[4] F. S. Tautz, Prog. Surf. Sci. 82, 479 (2007). 
[5] J. Hwang, A. Wan, and A. Kahn, Mater. Sci. Eng. 64, 1 (2009).

[6] R. Otero, J.M. Gallego, A. L. Vázquez de Parga, N. Martín, and R. Miranda, Adv. Mater. 23, 5148 (2011).

[7] A. Hauschild, K. Karki, B. C. C. Cowie, M. Rohlfing, F. S. Tautz, and M. Sokolowski, Phys. Rev. Lett. 94, 036106 (2005).

[8] R. Rurali, N. Lorente, and P. Ordejón, Phys. Rev. Lett. 95, 209601 (2005).

[9] A. Hauschild, K. Karki, B. C. C. Cowie, M. Rohlfing, F. S. Tautz, and M. Sokolowski, Phys. Rev. Lett. 95, 209602 (2005).

[10] S. Henze, O. Bauer, T.-L. Lee, M. Sokolowski, and F. S. Tautz, Surf. Sci. 601, 1566 (2007).

[11] L. Kilian, A. Hauschild, R. Temirov, S. Soubatch, A. Schöll, A. Bendounan, F. Reinert, T.-L. Lee, F. S. Tautz, M. Sokolowski et al., Phys. Rev. Lett. 100, 136103 (2008).

[12] M. Rohlfing and T. Bredow, Phys. Rev. Lett. 101, 266106 (2008).

[13] A. Abbasi and R. Scholz, J. Phys. Chem. C 113, 19897 (2009).

[14] M. Dion, H. Rydberg, E. Schröder, D. C. Langreth, and B. I. Lundqvist, Phys. Rev. Lett. 92, 246401 (2004).

[15] S. Grimme, J. Comput. Chem. 27, 1787 (2006).

[16] A. Tkatchenko, L. Romaner, O. T. Hofmann, E. Zojer, C. Ambrosch-Draxl, and M. Scheffler, MRS Bull. 35, 435 (2010).

[17] S. Grimme, WIREs Comput. Mol. Sci. 1, 211 (2011).

[18] L. Romaner, D. Nabok, P. Puschnig, E. Zojer, and C. Ambrosch-Draxl, New J. Phys. 11, 053010 (2009).

[19] F. J. Giessibl, Rev. Mod. Phys. 75, 949 (2003).

[20] N. Fournier, C. Wagner, C. Weiss, R. Temirov, and F. S. Tautz, Phys. Rev. B 84, 035435 (2011).

[21] R. Temirov, A. Lassise, F. B. Anders, and F. S. Tautz, Nanotechnology 19, 065401 (2008).
[22] C. Toher, R. Temirov, A. Greuling, F. Pump, M. Kaczmarski, M. Rohlfing, G. Cuniberti, and F. S. Tautz, Phys. Rev. B 83, 155402 (2011).

[23] A. Greuling, M. Rohlfing, R. Temirov, F. S. Tautz, and F. B. Anders, Phys. Rev. B 84, 125413 (2011).

[24] G. H. Simon, M. Heyde, and H.-P. Rust, Nanotechnology 18, 255503 (2007).

[25] Since PTCDA is neutral, electrostatic image forces are neglected.

[26] M. Mura, A. Gulans, T. Thonhauser, and L. Kantorovich, Phys. Chem. Chem. Phys. 12, 4759 (2010).

[27] M. J. Frisch et al.Gaussian 03 (Gaussian Inc., Wallingford CT, 2004)

[28] See Supplemental Material at http://link.aps.org/ supplemental/10.1103/PhysRevLett.109.076102 for a detailed description of the method that was used to generate and assess the parameter sets $Q$ and $P$ for the intramolecular and the molecule-substrate interactions and an animation of the lifting process.

[29] The molecule-tip bond is a Morse potential $(D=1 \mathrm{eV}$, $r_{0}=2.2 \AA, a=2.0 \AA^{-1}$ ) between one $\mathrm{O}_{\text {carb }}$ atom and the tip apex atom. The potential depths $D$ has barely any influence on the simulation as long as the bond is strong enough to lift the molecule.

[30] F. J. Giessibl, Appl. Phys. Lett. 78, 123 (2001).

[31] The physical origin of $A_{1}, A_{2}$, and $B$ is discussed in Ref. [20]. Our simulations show that the splitting of $A$ into $A_{1}$ and $A_{2}$ that is observable in the present experiments arises from the lifting of the first and second naphthalene unit of PTCDA.

[32] J. Ziroff, P. Gold, A. Bendounan, F. Forster, and F. Reinert, Surf. Sci. 603, 354 (2009).

[33] V. G. Ruiz, W. Liu, E. Zojer, M. Scheffler, and A. Tkatchenko, Phys. Rev. Lett. 108, 146103 (2012). 